\title{
La derecha invertida: Técnica y metodología
}

\author{
Encarna Martín-Lorente, José Campos y Miguel Crespo
}

\section{RESUMEN}

Este artículo estudia los aspectos técnicos y metodológicos de la derecha invertida. Por lo que hace referencia a la técnica, se enfatiza la importancia de un adecuado juego de pies para la ejecución eficiente de este gesto. En cuanto a las consideraciones metodológicas se sugiere la utilización de estrategias de descubrimiento. Finalmente se presentan algunos ejercicios en cancha para la mejora de la técnica del juego de pies.
Palabras clave: golpe de fondo, biomecánica, derecha

Recibido: 12 Abril 2017

Aceptado: 10 Junio2018

Autor correspondiente:

Encarna Martín-Lorente.

Correo electrónico:

encarna.martinlorente@gmail.com

\section{INTRODUCCIÓN}

La derecha invertida, se realiza cuando el jugador se desplaza sobre la mitad izquierda de la pista, con el objetivo inicial de resguardar el revés para emplear el golpe de derecha (Figura 1). En el caso de jugadores zurdos el desplazamiento se produce sobre el lado derecho

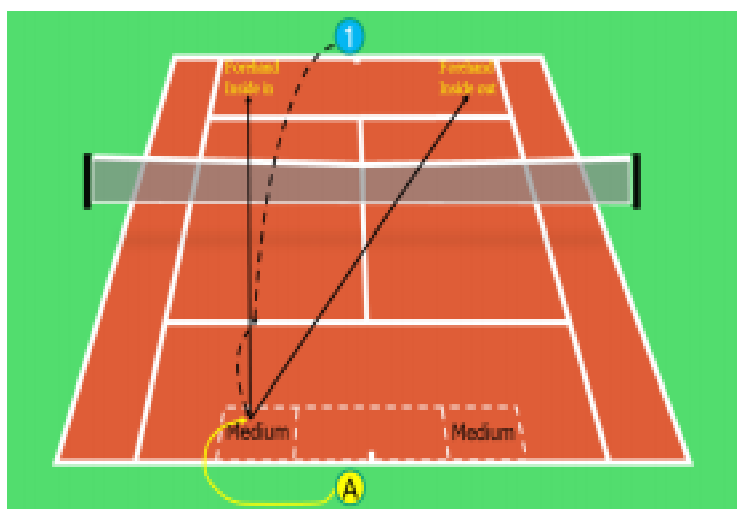

Figura 1. Derecha invertida de dentro a dentro y de dentro afuera.

La dirección de la pelota tras ser golpeada, da nombre al gesto técnico. Así, en el caso de la derecha invertida en dirección exterior o diagonal se denomina "derecha invertida hacia fuera" (forehand inside out"). Por contra, en el caso de emplear el gesto en dirección paralela se denomina "derecha invertida hacia dentro" ("forehand inside in").

La derecha invertida cruzada es el golpe 'dentro' más usado, porque pasa por la parte más baja de la red, sumando en seguridad; la pelota tiene mayor recorrido por ser un tiro diagonal, obligando a desplazar al oponente de la pista y generando espacio, disponiendo, a priori, de una buena posición de ataque. Con este golpe, por lo general, se busca "encerrar" al adversario por el lado del revés y luego buscar el tiro ganador al mismo lugar o el cambio hacia el otro lado de la pista.

La derecha invertida en dirección paralela es un golpe de mayor riesgo, ya que por lo general encuentra el drive del rival, y necesita de una muy buena velocidad y colocación (salvo que ya se haya generado el espacio previamente). Este golpe se ejecuta con un poco más de efecto que el cruzado, ya que la red está más alta y la pelota tiene menos recorrido.

\section{LA TÉCNICA Y EL JUEGO DE PIE DE LA DERECHA INVERTIDA}

En general, se trata de un gesto técnico de golpeo con la derecha, en el que la posición del cuerpo está condicionada por la disposición de los pies que se encuentran abiertos y situados lateralmente, permitiendo golpear la pelota con un ángulo más abierto, sobre el lado izquierdo de la pista, o sobre el lado derecho de la pista en caso de que el jugador sea zurdo como Nadal (Figura 2).

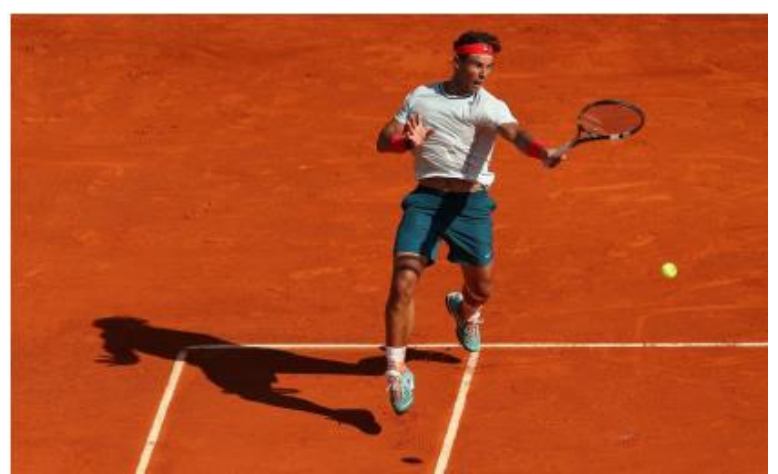




\section{Figura 2. Rafael Nodal.}

Como afirman Reid, Crespo, Santilli y Miley (2005) los desplazamientos cercanos son aquellos que requieren que un jugador se desplace hasta aproximadamente 3 metros como máximo para realizar un golpe. Por tanto, somos de la opinión que el juego de pies de la derecha escorada es de este tipo y, además, es crucial.

Así, al evaluar los patrones de desplazamiento de jugadores profesionales sobre tierra batida, Ferrauti y Weber (2001) observaron que aproximadamente el $80 \%$ de todos los golpes se juegan a una distancia menor de 3 metros desde la posición de preparado del jugador en el fondo de la pista. Para jugar de manera óptima golpes defensivos y ofensivos en desplazamientos cortos se necesita coordinación y agilidad, además de un equilibrio adecuado y versátil (Bourquin, 2003). Los desplazamientos cercanos básicos son los pasos de flotación ("shuffle") multidireccionales y el paso lateral, así como los pequeños pasos de ajuste.

Cabe destacar el desplazamiento lateral, rodeando la pelota para lograr el perfil adecuado, llegar a la misma con pasos cortos e ir haciendo el armado del golpe mientras se rodea la pelota, señalar con la mano izquierda para calcular la distancia con la pelota y tirar el peso del cuerpo hacia adelante en el momento del impacto para generar mayor potencia en el golpe.

Con respecto al trabajo específico del juego de pies en la derecha invertida, Reid et al., (2005) consideran que los ejercicios de juego de pies que trabajan este aspecto tan importante del desplazamiento y, por tanto, de la ejecución de los golpes han jugado, durante los últimos 15 años, un papel fundamental en el entrenamiento en pista de muchos profesionales. Pato Álvarez, uno de los más destacados entrenadores de España, utilizó habitualmente muchos de estos ejercicios con sus jugadores en la década de los 80 y 90 , tanto que se han convertido en un elemento característico del repertorio de muchos entrenadores de alto nivel.

En este sentido, estos mismos autores creen que con la proliferación en los últimos años de jugadores de fondo de pista muy potentes, la capacidad de llegar con velocidad y eficacia a pelotas anguladas cerca de las líneas laterales y de recuperarse, tiene cada vez más importancia. La habilidad de cubrir la pista junto con unos desplazamientos efectivos en las distancias cortas forma el repertorio de juego de pies de la mayoría de los jugadores profesionales.

La comparación entre jugadores iniciantes y avanzados en la preparación para un golpe de fondo en carrera ilustra un elemento importante del desplazamiento que debe entrenarse en la pista (Saviano, 2000). En los jugadores avanzados el desplazamiento hacia un golpe de fondo se inicia en el tren inferior y la fase inicial de la rotación del hombro se produce sin mover excesivamente la raqueta (Ellenbecker y Roetert, 2003). Esto permite al jugador iniciar la rotación del tronco sin que la raqueta le moleste para moverse hacia la pelota. Por el contrario, en los mismos golpes, los iniciantes a menudo se preparan y salen corriendo hacia la pelota con el brazo y la raqueta totalmente extendidos detrás de la espalda.

Independientemente del nivel de juego, durante los desplazamientos en la pista es fundamental que los jugadores adopten una postura equilibrada para transferir la fuerza de la manera más eficiente posible y proporcionar una base estable (para la cabeza) desde la que procesar la información visual adecuadamente. Es importante entrenar especialmente el mantenimiento de una postura corporal correcta durante los desplazamientos en la pista y la ejecución de los golpes
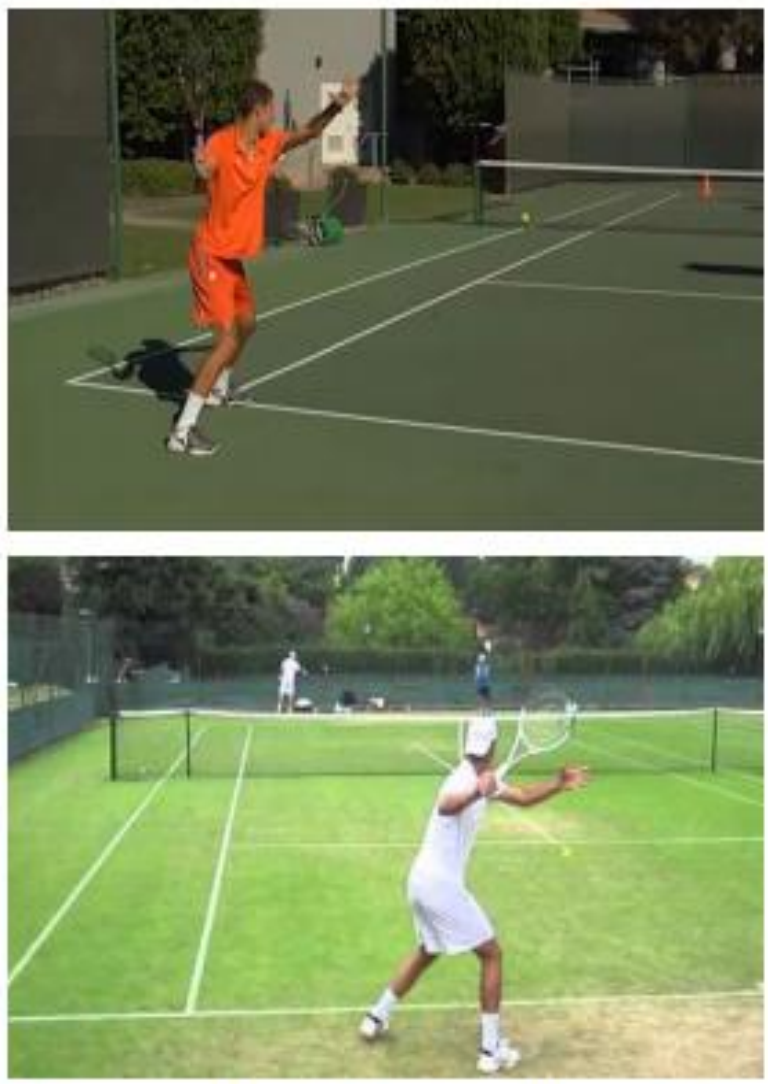

(Ellenbecker y Roetert, 2003; Verstegen, 2003) (Figuras 3 y 4 ).

\section{LA ENSEÑANZA DE LA DERCHA INVERTIDA: CRITERIOS METODOLÓGICOS}

Brabenec (1996) afirma que en el tenis moderno, el golpe de derecha debe ser un "arma" (hay que cubrir de un 65 a un $70 \%$ de la pista con la derecha) y el revés un sólido complemento de apoyo. Además, Cuando el contrario juega una pelota lenta dirigida a nuestro revés, hay que "rodear" el revés siempre que sea posible para jugar una derecha de ataque. La derecha jugada desde el rincón del revés permite "camuflar" las intenciones del jugador que golpea la pelota. Puede jugarse una derecha de dentro hacia afuera o paralela a la línea lateral. También nos ofrece una mayor probabilidad de jugar otro golpe de derecha, en caso de que la pelota sea devuelta por el contrario. 
Para Dent (1996) el resto de segundo saque con una derecha escorada es una opción excelente para ser agresivo en este golpe. El entrenador o un jugador dirigen un segundo saque al lado del revés del adversario. El restador "rodea" su revés para restar con una derecha de ataque.

Así, el restador debe moverse para "rodear el revés" cuando el jugador que sirve ha efectuado la elevación de pelota. El resto de mayor "porcentaje" es un golpe de derecha cruzado de dentro hacia afuera, ya que ello obliga al jugador que ha efectuado el saque a cambiar de dirección. Es la mayor distancia sobre la parte más baja de la red y permite al restador desplazarse fácilmente para volver a la posición geométrica central, reduciendo al mínimo los ángulos de ataque del contrario

Farrell (1998) cuando habla de la mecánica del juego de pies para hacer frente a la pelota corta sugiere que muchos jugadores preferirán evitar su revés y jugar una derecha escorada y que, por tanto, el entrenador debe enseñar y entrenar este movimiento.

Por otro lado, hay que fomentar en el jugador la sensación de "pegar al máximo" hacia la línea de fondo lejana, de forma que el hombro y la raqueta se desplacen hacia adelante a la misma altura que el punto de contacto (si el punto de contacto se halla a la altura del hombro, como pasará invariablemente).

\section{CONCLUSIONES Y APLICACIONES PRÁCTICA}

En la enseñanza y el entrenamiento de la técnica de la derecho invertida es recomendable utilizar la estrategia didáctica de descubrimiento guiado o de resolución de problemas en la que el entrenador genere situaciones de juego que planteen retos al jugador en los que este tenga que descubrir la solución. En este caso, esto se busca mediante el enfoque de enseñanza con constreñimientos y haciendo uso de una gran variabilidad en la práctica (Martín-Lorente, Campos, \& Crespo, 2017).

Los enfoques abiertos, globales, holísticos, facilitadores de soluciones adaptadas, que impliquen un conocimiento implícito por parte del tenista y una comprensión de las exigencias del juego son, obviamente, los más adecuados.

\section{Ejercicio 1}

Objetivo: Trabajo del juego de pies específico para la derecha invertida.

Lugar y material: Pista de tenis, raqueta, diana.

Metodología: Sombras de movimiento sin pelota.

Descripción: El jugador se coloca aproximadamente 1 metro tras la marca central de servicio al fondo de la pista. Se coloca un cono tal y como indica la figura. El jugador sujetando la raqueta se desplazará realizando el movimiento para golpear de derecha invertida y ejecutará el gesto del golpe (Figura 5).

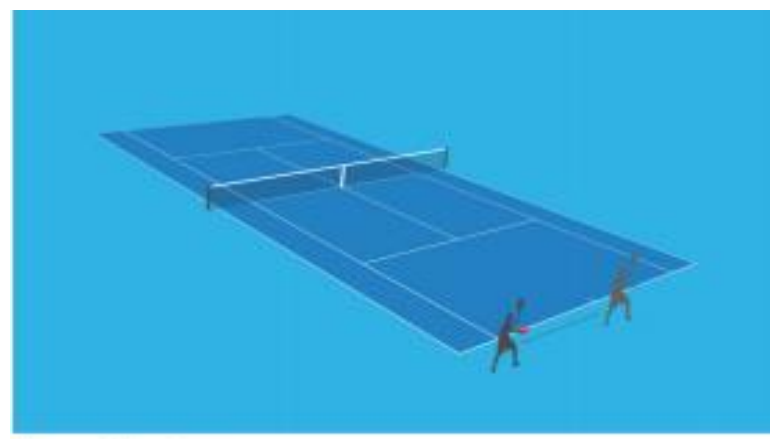

Figura 5. Ejercicio 1.

Ejercicio 2

Objetivo: Trabajo del juego de pies específico y el golpeo de la derecha invertida.

Lugar y material: Pista de tenis, cesto de pelotas, raqueta, dianas.

Metodología: Lanzamiento del entrenador con la mano.

Descripción: El jugador se sitúa aproximadamente un metro tras la marca central de servicio en la zona izquierda de la pista. Se coloca un cono tal y como indica la figura. El entrenador se coloca aproximadamente a mitad del pasillo de dobles del lado izquierdo de la pista y lanza las pelotas con la mano a la zona indicada en la figura para que el jugador realice el movimiento hacia la pelota con el juego de pies adecuado tal y como demuestran la flecha negra. El jugador golpeará de derecha invertida hacia las dianas fijadas en la otra media pista (Figura 6)

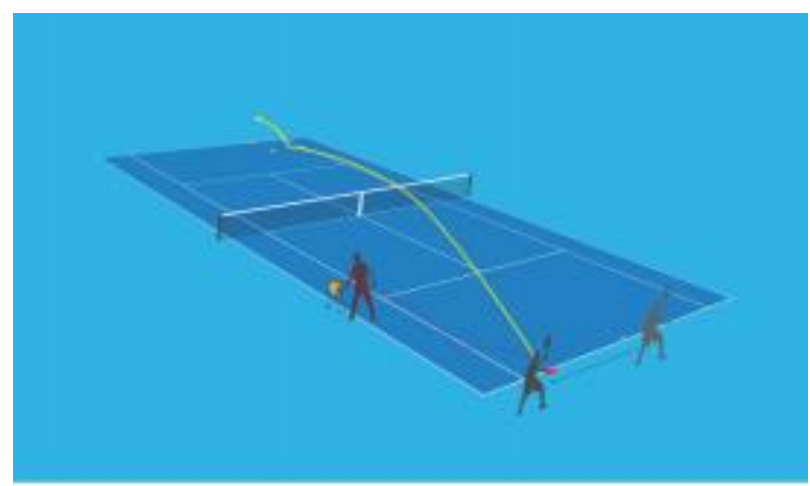

Figura 6. Ejercicio 2.

Ejercicio 3

Objetivo: Trabajo del juego de pies específico, el golpeo y la dirección del golpe de derecha invertida en una situación cerrada.

Lugar y material: Pista de tenis, cesto de pelotas, raqueta, dianas.

Metodología: Lanzamiento del entrenador con la cesta.

Descripción: Similar al ejercicio anterior pero el entrenador situado en la otra mitad de la pista lanza las pelotas desde el 
cesto para que el jugador realice el movimiento hacia la pelota con el juego de pies adecuado tal y como demuestran la flecha. El jugador golpeará de derecha invertida hacia las dianas fijadas en la otra media pista alternando la dirección de los golpes (Figura 7).

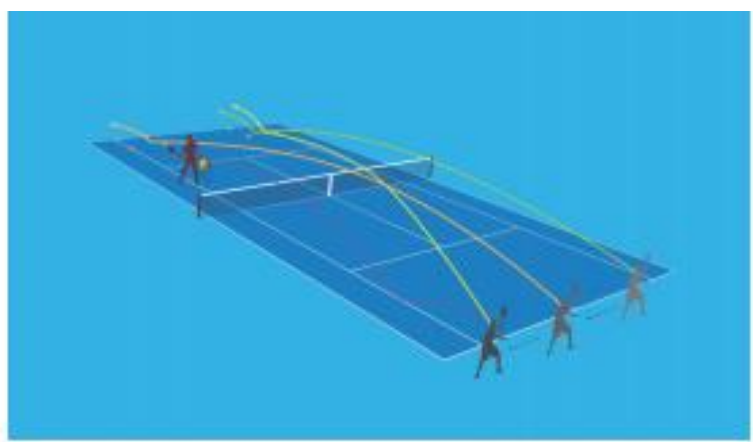

Figura 7. Ejercicio 3.

\section{Ejercicio 4}

Objetivo: Trabajo del juego de pies específico, el golpeo y la dirección del golpe de derecha invertida en situación semi cerrada.

Lugar y material: Pista de tenis, cesto de pelotas, raqueta, dianas.

Metodología: Lanzamiento del entrenador con la cesta.

Descripción: Similar al ejercicio anterior pero el entrenador situado en la otra mitad de la pista lanza las pelotas desde el cesto para que el jugador realice el movimiento hacia la pelota con el juego de pies adecuado tal y como demuestran las flechas negras. El entrenador lanza tres pelotas por serie. Una a la zona izquierda del jugador, una al centro y otra a la zona derecha. No es necesario colocar un cono en el lado de la pista del jugador para evitar que tropiece. El jugador golpeará todas las pelotas de derecha invertida hacia las dianas fijadas en la otra mitad de la pista alternando la dirección de los golpes (Figura 8).

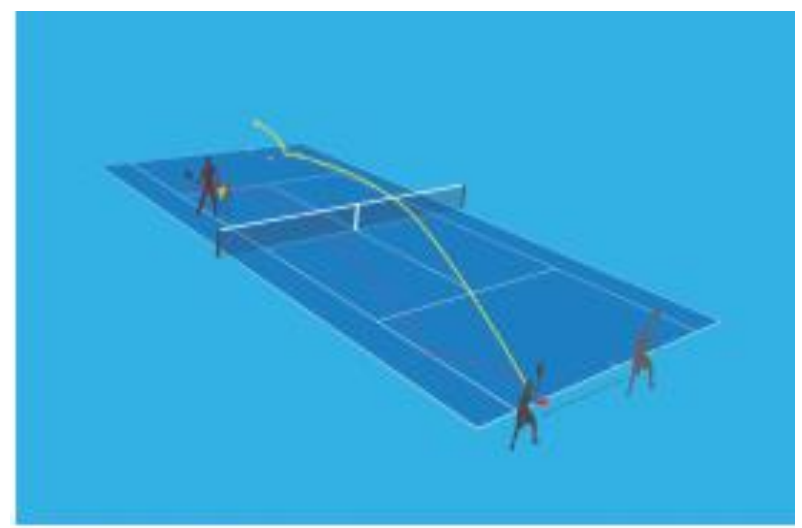

Figura 8. Ejercicio 4 .

\section{REFERENCIAS}

Bourquin, O. (2003). Coordination. In M. Reid, A. Quinn, M. Crespo (Eds.). Strength and Conditioning for Tennis. Londres: International Tennis Federation

Brabenec, J. (1996). If a player knows how, then when is the important question, ITF Coaching and Sport Science Review, 10, 7-8.

Dent, P. (1996). Coordinate to accelerate. ITF Coaching and Sport Science Review, 8.

Ellenbecker, T., \& Roetert, E. P. (2003). Age specific isokinetic glenohumeral internal and external rotation strength in elite junior tennis players. Journal of science and medicine in sport, 6(1), 63-70. 26 Año, Número 76, Diciembre 20189 https://doi.org/10.1016/S1440-2440(03)80009-9

Farrell, P. (1998). The short ball, ITF Coaching and Sport Science Review, 16, 4

Martín-Lorente, E.; Campos, J.; \& Crespo, M. (2017). The inside out forehand as a tactical pattern in men's professional tennis, International Journal of Performance Analysis in Sport, Vol. 17, Iss. 4. https://doi.org/10.1080/24748668.2017.1349528

Martín-Lorente, E.; Campos, J.; \& Crespo, M. (2018). The inside out stroke in men's tennis: strategies and tactics, ITF CSSR, 74, 26, 20 $-22$

Reid, M., Crespo, M., Santilli, L. \& Miley, D. (2005). The ITF Junior Boys' Circuit and its Role in Professional Player Development, ITF Coaching and Sport Science Review, 35.

Saviano, N. (2000). Dispelling Technical Myths: The Split Step and Racquet Preparation. High Performance Coaching, United States Tennis Association.

Verstegen, M. (2003). Developing Strength. In: Strength and Conditioning for Tennis, International Tennis Federation, 2003, 114-135.

CONTENIDO ITF ACADEMY RECOMENDADO (HAZ CLICK ABAJO)

\section{ITF Academy}

Derechos de Autor (c) 2018 Encarna Martín-Lorente, José Campos y Miguel Crespo

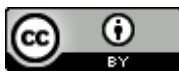

Este texto está protegido por una licencia CreativeCommons 4.0.

Usted es libre para Compartir —copiar y redistribuir el material en cualquier medio o formato-y Adaptar el documento - remezclar, transformar y crear a partir del material- para cualquier propósito, , incluso para fines comerciales, siempre que cumpla la condición de:

Atribución: Usted debe dar crédito a la obra original de manera adecuada, proporcionar un enlace a la licencia, e indicar si se han realizado cambios. Puede hacerlo en cualquier forma razonable, pero no de forma tal que sugiera que tiene el apoyo del licenciante o lo recibe por el uso que hace de la obra.

Resumendelicencia - Textocompletodelalicencia 